\title{
Epigenetics, Histone H3 Variants, and the Inheritance of Chromatin States
}

\author{
S. Henikoff, ${ }^{*}$ E. McKitTrick, ${ }^{*}$ And K. Ahmad ${ }^{\dagger}$ \\ ${ }^{*}$ Howard Hughes Medical Institute, Fred Hutchinson Cancer Research Center, Seattle, Washington 98109; \\ ${ }^{\dagger}$ Department of Biological Chemistry and Molecular Pharmacology, Harvard Medical School, Boston, \\ Massachusetts 02115
}

The 68th Cold Spring Harbor Symposium celebrated an extraordinary accomplishment in molecular biology: determination of the human genomic sequence. This landmark achievement highlights the rapid and steady progress that has occurred over the past 50 years beginning with the Watson-Crick model of DNA and culminating in the nearly complete specification of our genetic inheritance. In contrast, the 69th Symposium deals with a topic, epigenetics, that was already 25 years old at the time of the Watson-Crick model, and yet the salient features of epigenetic phenomena have remained almost as mysterious now as when they were first described.

The experimental study of epigenetics began when H.J. Muller discovered a remarkable class of mutations that appeared following X-ray irradiation of flies (Muller 1930). This phenomenon, called position-effect variegation (PEV), is the unstable expression of a gene that results when chromosome breaks juxtapose it to heterochromatin, the cytologically condensed regions of chromosomes (Spofford 1976). Although Muller first observed the phenomenon in rearrangements that affect the white eye color gene, similar effects have been found for many other types of genes and in organisms ranging from animals to fungi to plants (Richards and Elgin 2002).

Three striking features that are apparent from PEV phenotypes continue to intrigue us today. First, PEV appears to be an all-or-none phenomenon. This is readily apparent in a modern example of classical PEV seen in Figure 1, which shows a fly that harbors a variegating allele of a green fluorescent protein (GFP) gene that had been juxtaposed to heterochromatin by a chromosomal rearrangement. The gene shows its normal level of expression in some tissues of the eye, but appears to be completely inactive in other tissues. Second, inactivation is patchy, as though a decision was made as to whether or not to be active, and that decision was inherited through multiple rounds of cell division. Finally, the patterns of expression are completely different between the two eyes of this fly, as if such decisions could occur essentially at random throughout development. These three features, all-or-none expression, heritability, and random decisions between states, are characteristic of epigenetic phenomena in general.

We have attempted to understand these prominent fea- tures of PEV by applying modern visualization tools and reporters to the problem. We first describe the use of a Drosophila genetic system for probing the accessibility of a promoter that is subject to PEV (Ahmad and Henikoff 2001b). This experimental system has provided insights into the all-or-none and stochastic nature of PEV. Next, we describe a Drosophila cytological system for observing dynamic chromatin changes during the cell cycle that might be involved in epigenetic phenomena (Ahmad and Henikoff 2001a). We have applied this system to the study of variant $\mathrm{H} 3$ histones, where we have uncovered a distinct pathway for nucleosome assembly that takes place at active genes (Ahmad and Henikoff 2002c). This discovery motivated a biochemical approach to ascertain the relationship between $\mathrm{H} 3$ variants and their covalent modifications (McKittrick et al. 2004). We propose that nucleosome assembly pathways represent fundamental means of differentiating chromatin states that can account for precise inheritance of chromatin memory and PEV through multiple rounds of cell division.

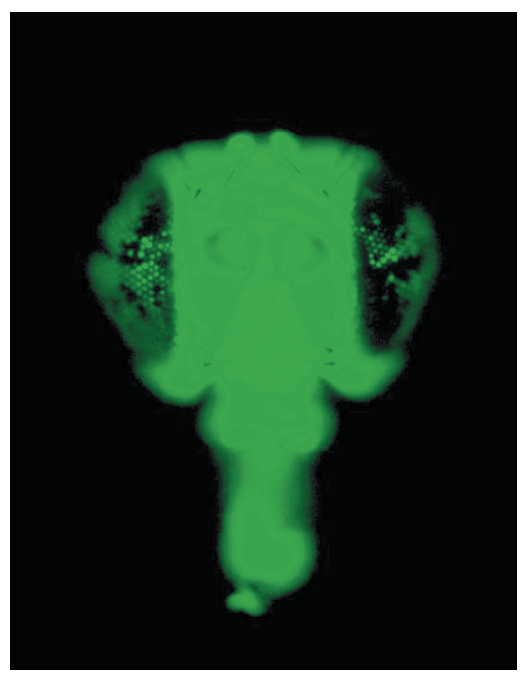

Figure 1. PEV illustrates features of epigenetic inheritance. Image of the head of a fruit fly, showing the stochastic epigenetic silencing of a transgene encoding GFP in individual ommatidia of the eyes. See text for details. 


\section{EPIGENETIC PHENOTYPES AND NUCLEOSOME DYNAMICS}

\section{Probing Heterochromatin during Drosophila Development}

To study PEV during development, we introduced a novel in vivo reporter system (Fig. 2A). Our starting line harbored a $P$ transposon that contained a set of five tandem yeast GAL4 binding sites upstream of a gene encoding GFP ( $U A S G F P$ ), inserted into a euchromatic site (Ahmad and Henikoff 2001b). The transposon also contained a mini-white eye color gene, which provided a reporter for selection of PEV mutants. After X-ray irradiation to induce chromosomal rearrangements, we screened for progeny that showed classical white variegation. We then subjected lines to $P$-element mobilization, which allowed us to select for more severe variegators (Dorer and Henikoff 1994). The PEV nature of these mutant lines was confirmed by showing that variegation was suppressed at elevated temperature and in the presence of a Su(var)205 mutation, a suppressor of PEV that encodes Heterochromatin-associated Protein 1 (HP1) (Weiler and Wakimoto 1995).

When two genes are present on a transposon and one is subject to PEV, it is generally observed that the other gene variegates as well. To test for variegation of the UASGFP gene in lines showing mini-white variegation, we crossed each line to flies that produce GAL4, which activates the $U A S G F P$ gene when bound to its upstream activation sequence (Ahmad and Henikoff 2001b). Expression was assayed in eye discs of third instar larvae. Using a "driver" $(G a w B)$ that expresses GAL4 beginning in the early larval period and continuing throughout development, we observed patchy expression relative to the uniform expression that is characteristic of wild-type, indicating PEV of UASGFP (Fig. 2B). Patches appeared clonal, often crossing the morphogenetic furrow that separates proliferating from differentiated cells as it moves from posterior to anterior of the disc. Interestingly, silencing in the same $U A S$ GFP lines was less pronounced in the presence of GAL4 produced by a driver $(A 5 C)$ that began to express during early embryonic development, and silencing was more severe using a driver (GMR) that expressed behind the morphogenetic furrow during pupal development (Fig. 2B). Such differences could not be accounted for by differences in the amount of GAL4 produced, because these levels were estimated to be highest in tissues expressing GAL4 only in differentiated cells $(G M R)$, which showed the strongest degree of silencing (Fig. 2B).

\section{Activation Depends on Transcription Factor Abundance}

A simple explanation for different degrees of silencing produced by drivers that turn on at different times during development is that heterochromatin usually, but not always, prevents GAL4 from gaining access to its binding site. However, once GAL4 binds, then the gene remains active throughout the remainder of development. In this way, the longer that GAL4 is available in developing tis- sue, the higher the probability that it will bind and permanently activate expression. This hypothesis predicts that the probability of expression will also be increased by higher concentrations of GAL4. Indeed, this is the case; we found that two drivers that begin expression at the same early time in development but at approximately tenfold different levels ( $A 5 C$ and $\mathrm{arm}$ ) show corresponding differences in the probability of activation (Fig. 2B). Further support for this hypothesis came from the detection of spontaneous activation in cultured discs on both sides of the morphogenetic furrow (Ahmad and Henikoff 2001b). These observations imply that heterochromatin is in a dynamic state, because yeast GAL4, which can act in Drosophila only once it is bound, appears to gain access to its binding site in heterochromatin in a stochastic manner at any time during development.

Our conclusion that heterochromatin is dynamic (Ahmad and Henikoff 2001b, 2002a) later received strong support from fluorescence recovery after photobleaching
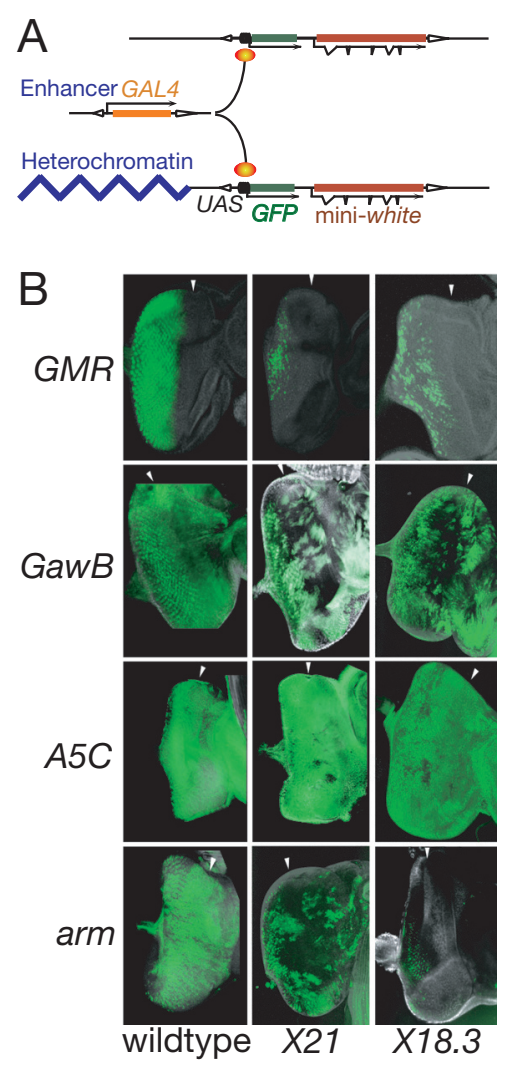

Figure 2. Dependence of PEV on transcription factor concentration (Polach and Widom 1995). (A) Schematic diagram of a system for probing heterochromatin during Drosophila development. GAL4 controls expression of the UAS-GFP reporter gene located either in its normal position (top) or juxtaposed to constitutive heterochromatin (bottom). (B) Eye-antennal imaginal discs expressing $U A S-G F P$ under control of GAL4 driven by the GMR eye- and differentiation-specific enhancer ( $G M R)$, by the GawBT80 larval enhancer $(G a w B)$, by the strong Actin5C $(A 5 C)$ embryonic enhancer, and by the weak armadillo (arm) embryonic enhancer. The reporter gene is on a wild-type chromosome (left column) and is subject to PEV in two different lines (center and right column). Arrowheads, the location of the morphogenetic furrow. 
studies of the key component of heterochromatin, HP1 (Cheutin et al. 2003; Festenstein et al. 2003). HP1 associates with chromatin with a residence time of only several seconds. The dynamic nature of heterochromatin is surprising given the perception that it reflects a condensed state, indicating the need to consider dynamic models of heterochromatin to explain PEV. An attractive possibility comes from a proposal by Widom and colleagues based on their in vitro studies of the accessibility of a DNAbinding site when it is wrapped up in a nucleosome (Polach and Widom 1995). They hypothesized that an equilibrium exists between a conformation in which a binding site is inaccessible because it is wrapped and a conformation in which the site is accessible because it is unwrapped. We expect that in the wild-type euchromatic situation, this equilibrium would not be directly observable, because the unwrapped conformation would also be in equilibrium with an unwrapped factor-bound conformation, and this bound state would be strongly favored (Fig. $3)$. However, if juxtaposition to heterochromatin increases the frequency of a nucleosome being wrapped, then there will be two relatively stable conformations (wrapped and factor-bound) separated by a transient unwrapped and unbound intermediate (Ahmad and Henikoff 2001b). The existence of a distinct intermediate state is crucial, because in a two-conformation competition model (Dillon and Festenstein 2002), each cell should show a time-averaged intermediate level of expression, not stochastic variegation.

Our dynamic equilibrium model fits with several known features of heterochromatin and PEV. HP1 is a dimer with two chromodomains that bind to methylated histone $\mathrm{H} 3$ lysine-9 (H3K9me), a modification that is especially abundant in heterochromatin (Jenuwein and Allis 2001). H3 tails exit between the DNA gyres on either side of the nucleosomal dyad axis and associate with linker histones and linker DNA (Luger et al. 1997; Zheng and Hayes 2003). Therefore, it is expected that the effect of HP1 binding, even briefly, to pairs of H3 tails would be

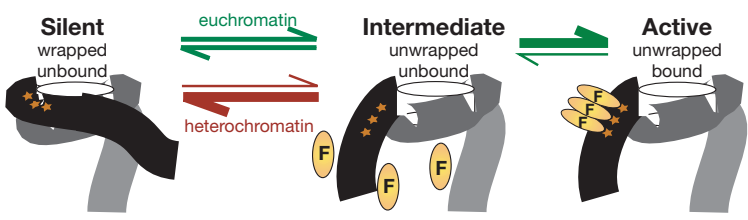

Figure 3. A site-exposure model for gene silencing (Ahmad and Henikoff 2001b). When DNA containing factor-binding sites (stars) is wrapped around a nucleosome, the sites are inaccessible. Transient unwrapping of the DNA exposes the site, which can then be bound by factor $(\mathrm{F})$. Let $[\mathrm{S}]=$ the concentration of silent nucleosomes, $[\mathrm{A}]=$ the concentration of active nucleosomes, $[\mathrm{I}]=$ the concentration of the transient intermediate, and $[\mathrm{F}]=$ the concentration of factor. Define rate constants as $k_{1}$ $(\mathrm{S} \rightarrow \mathrm{I}), k_{2}(\mathrm{I} \rightarrow \mathrm{S}), k_{3}(\mathrm{~A} \rightarrow \mathrm{I}+\mathrm{F})$, and $k_{4}(\mathrm{I}+\mathrm{F} \rightarrow \mathrm{A})$. Then $d[\mathrm{~S}] / d t=$ $-k_{1}[\mathrm{~S}]+k_{2}[\mathrm{I}]$, and $d[\mathrm{~A}] / d t=-k_{3}[\mathrm{~A}]+k_{4}[\mathrm{I}][\mathrm{F}]$. At equilibrium, $d[\mathrm{~S}] / d t=d[\mathrm{~A}] / d t=0$, so $[\mathrm{I}]=\left(k_{1} / k_{2}\right)[\mathrm{S}]=\left(k_{3} / k_{4}\right)[\mathrm{A}] /[\mathrm{F}]$, or $[\mathrm{A}] /[\mathrm{S}]=\left(k_{1} k_{4} / k_{2} k_{3}\right)[\mathrm{F}]$. That is, the ratio of active to silent tissue should be directly proportional to transcription factor concentration. (Reprinted, with permission, from Ahmad and Henikoff 2001b [@Elsevier].) to reduce the frequency of unwrapping, thus disfavoring intermediate formation and reducing the availability of factor-binding sites. The model also can explain the dependence of activation frequency on transcription factor concentration observed in our in vivo assay (Fig. 2B); examination of the equilibrium equations reveals that the ratio of active to silent tissue should be directly proportional to the concentration of transcription factor (see the Fig. 3 legend). Finally, the model provides an intuitive explanation for both the on-off and the stochastic nature of PEV. So, although PEV is notorious as a long-range effect, the ultimate nature of silencing (but not "spreading") is a process that may be understood in molecular detail at the level of a single nucleosome.

\section{NUCLEOSOME ASSEMBLY PATHWAYS DISTINGUISH CHROMATIN STATES}

\section{How Are Chromatin States Inherited?}

The dynamic nature of heterochromatin makes the problem of propagating the heterochromatic state through rounds of cell division seem even more mysterious. If the silent state is unstable, with HP1 and other chromatin-associated proteins rapidly exchanging, why is PEV so often clonal? This question of epigenetic memory is a general one that appears to underlie most cases of developmental decisions that are remembered through multiple rounds of cell division.

One possible way that epigenetic memory might be inherited is by replication of histone modifications. This possibility has generated much excitement in the chromatin field in recent years with the realization that active and silent chromatin are differentially modified by enzymes that have been implicated in the specification of chromatin states (Jenuwein and Allis 2001; Grewal and Elgin 2002). Furthermore, the binding of HP1 to H3K9me provides a direct link between this particular histone modification and the heterochromatic state. However, the process whereby modifications are replicated has remained unclear. This is a serious problem, because old nucleosomes are randomly distributed intact behind the replication fork (Leffak et al. 1977; Yamasu and Senshu 1990), leading to dilution of epigenetic signals by a factor of 2 and loss of precision with each cell division. Chromatin inheritance appears to be unlike DNA methylation, which is maintained by the Dnmt1 DNA methyltransferase with base-pair precision behind the replication fork (Leonhardt et al. 1992). Thus far, no mechanism is known that can replicate modifications on histones following DNA replication.

Our work on histone variants provides a potential solution to this enigma. We have discovered that a histone 3 variant, H3.3, replaces canonical $\mathrm{H} 3$ at active loci via a distinct pathway of replication-independent nucleosome assembly (Ahmad and Henikoff 2002c). The fact that H3 is not used by this pathway provides a distinction between active and inactive chromatin states. Replicationindependent deposition may serve to replenish a histone mark by replacing histones at active genes throughout the cell cycle. 


\section{A Cytological Assay Reveals Alternative Nucleosome Assembly Pathways}

To study changes that occur in nuclei during the cell cycle, we took advantage of the Drosophila Kc cell line in which the difference between early replicating euchromatin and late replicating heterochromatin is especially pronounced (Ahmad and Henikoff 2001a). Brief pulses of nucleoside triphosphates or probing with an antibody against a component of the DNA replication apparatus provides a snapshot of replication forks at the time that cells are fixed for cytological observations. The formation of a chromocenter by the coalescence of all heterochromatin in a cell reveals a clear spatial distinction between early and late replication, thus identifying cell cycle stages in an unsynchronized population. To follow the behavior of particular nuclear proteins during the cell cycle, we constructed plasmids encoding fusions to GFP under control of a heat shock promoter, introduced the plasmids into $K c$ cells by transient transfection, and produced chimeric protein with a heat shock pulse.

When this cytological assay system was applied to the centromere-specific $\mathrm{H} 3$ variant, CID, we were surprised to find that CID-GFP localized to centromeres throughout the cell cycle, indicating replication-independent (RI) assembly (Ahmad and Henikoff 2001a). Similar conclusions were reached by Sullivan and coworkers (Shelby et al. 2000) for CENP-A, the human centromere-specific $\mathrm{H} 3$ variant. In contrast, canonical $\mathrm{H} 3$ only deposited at replication forks in our assay (Ahmad and Henikoff 2001a).

A control experiment led us to realize that there is a distinct RI pathway for nucleosome assembly that acts at active loci. We had expected that histone H4-GFP would deposit at all sites of H3 and CID localization in gap phase cells when produced in the same assay. It did, but it also localized elsewhere (Ahmad and Henikoff 2002c). By far the most prominent site of RI deposition of H4GFP was an rDNA array (confirmed by fluorescence in situ hybridization using an rDNA probe), with weaker labeling throughout the chromosome arms, but not in heterochromatin. Thus, H4, but not $\mathrm{H} 3$, is deposited during gap phase at particular loci.

$\mathrm{H} 3$ and $\mathrm{H} 4$ are folded together by histone chaperones upon synthesis and are always deposited as a unit (Mello and Almouzni 2001), so we reasoned that the partner for $\mathrm{H} 4$ deposition at particular loci must be the replacement variant H3.3. Indeed, like $\mathrm{H} 4, \mathrm{H} 3.3$ was deposited in a replication-coupled (RC) manner during $\mathrm{S}$ phase and localized to an rDNA array and euchromatin during gap phases (Fig. 4) (Ahmad and Henikoff 2002c). This differential behavior of H3 and H3.3 was unexpected, insofar as the RI form of histone 3 in Tetrahymena was shown to be interchangeable with the $\mathrm{RC}$ form ( $\mathrm{Yu}$ and Gorovsky 1997). It was assumed that $\mathrm{H} 3$ and $\mathrm{H} 3.3$ would be likewise interchangeable, with only four amino acid differences between them. However, these differences are responsible for completely different deposition behavior of the histones when produced in the same way during gap phase (cf. Fig. 5A and Fig. 5B). The fact that $\mathrm{H} 3.3$ is $100 \%$ identical throughout its length in organisms

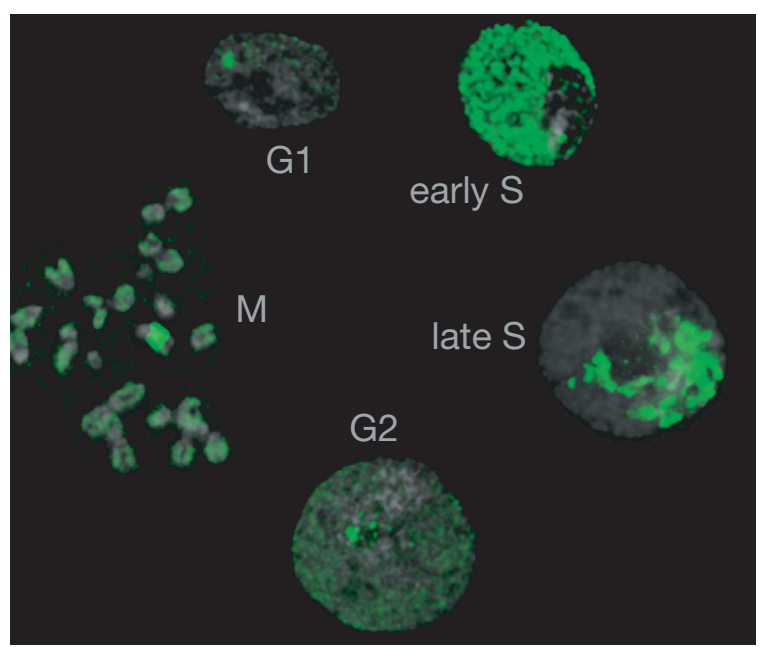

Figure 4. RC and RI deposition of H3.3 (Ahmad and Henikoff 2002c). Nuclei labeled with DAPI (gray) show the location of H3.3-GFP (green) when it is produced in a pulse at different times in the cell cycle. A mitotic figure is shown on the left (M), with $\mathrm{H} 3.3$ prominently deposited at the rDNA locus within $\mathrm{X}$ chromosome heterochromatin and throughout the euchromatic arms but not the heterochromatin. Proceeding clockwise are nuclei in G1 phase showing a bright rDNA focus, in early S phase showing RC euchromatic deposition, in late S phase showing $\mathrm{RC}$ heterochromatic deposition at the chromocenter, and in G2 phase showing bright rDNA foci and faint RI euchromatic deposition.

as diverse as flies, humans, and clams suggested that this difference in nucleosome assembly behavior is a general feature of animal chromatin, and a comparable situation appears to hold in plants (Waterborg 1993).

\section{Distinct Substrate Requirements for Alternative Nucleosome Assembly Pathways}

Three of the four amino acids that distinguish $\mathrm{H} 3$ from H3.3 are found in a cluster on the core (residues 87-90) and one lies on the amino-terminal tail (residue 31) (Malik and Henikoff 2003). Our gap phase incorporation assay revealed that $\mathrm{H} 3$ does not deposit into chromosomes at all when produced during G2 phase, but rather can be seen to accumulate throughout the cell at mitosis (Fig. 5B). Swapping these residues from H3.3 into H3 led to partial deposition of the GFP fusion at the rDNA array for each of the three core residues (Fig. 5C), but swapping the tail residue or swapping any single $\mathrm{H} 3$ residue into H3.3 had no effect. This suggests that something interacts with the three core residues of $\mathrm{H} 3$ and actively prevents its RI deposition.

Another sequence requirement that distinguishes RC from RI nucleosome assembly is a requirement for the amino-terminal tail. RC but not RI deposition of either $\mathrm{H} 3$ or H3.3 is gradually lost as more and more of the amino-terminal tail is removed (Ahmad and Henikoff 2002c). Although we do not understand the basis for this restriction, it is interesting that yeast lack this requirement, and, like other ascomycetes, yeast lack the H3 (RC) 

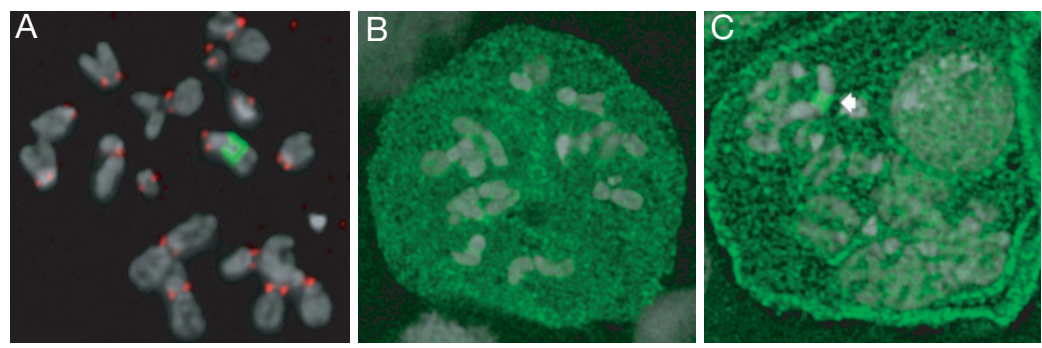

Figure 5. Mapping determinants of RC and RI assembly (Ahmad and Henikoff 2002c). (A) G2 phase incorporation of H3.3-GFP at the X-long rDNA array (green). Centromeres are marked with anti-CID antibody (red). (B) H3-GFP fails to incorporate into the chromosomes when produced during $\mathrm{G} 2$, and accumulates in the cell volume at mitosis. (C) Changing a single $\mathrm{H} 3$ amino acid residue on the core to the corresponding H3.3 residue (Met to Gly at position 90) causes partial incorporation of GFP at the rDNA (arrow). Similar results were obtained for the other two core residues (A87S and I89V), but no effect on incorporation was seen for the tail residue (A31S) or for any single change from H3.3 to H3. (Reprinted, with permission, from Ahmad and Henikoff 2002c [@Elsevier].)

form, having lost the canonical variant after splitting from basidiomycete fungi (Malik and Henikoff 2003). Thus, ascomycetes have only the H3.3 form, which is ironic considering that most of our knowledge of chromatin comes from studies in budding yeast, whereas almost all studies of higher eukaryotic chromatin have used the more abundant $\mathrm{H} 3$ form.

Our discovery of alternative chromatin assembly pathways based on substrate requirements has recently received direct biochemical support. Using affinity purification of tagged H3 and H3.3, Tagami et al. (2004) have found that soluble $\mathrm{H} 3$ is present in a complex with the RC-specific chromatin assembly factor 1 (CAF-1), and soluble H3.3 with the RI-specific histone regulator A (HIRA).

\section{H3.3 Replaces H3K9me at Epigenetically Activated Loci}

In tetraploid Drosophila Kc cells, RI assembly of H3.3 typically occurs at only one rDNA array (Ahmad and Henikoff 2002c), despite the presence of multiple X chromosomes, each with a single rDNA array. The X chromosomes in $K c$ cells are of two different types based on the size of their rDNA arrays (Echalier 1997). One X chromosome has undergone a large expansion of the rDNA locus relative to wild type, evidently an adaptation to culture conditions after the lines were established. We noticed that in quiescent cells, this "X-long" chromosome was always labeled with H3.3-GFP when produced during G2 (Fig. 6A). The absence of RI deposition of H3.3GFP at "X-short" does not appear to result from any permanent defect in X-short, because when cells are induced to grow, about half of them show RI deposition of H3.3GFP at the rDNA locus on at least one of the X-short chromosomes (Fig. 6B). This epigenetic change in an rDNA array is reminiscent of the well-known phenomenon of nucleolar dominance, where the rDNA array from one species in an interspecific hybrid is active while that from the other species is inactive (Pikaard 2000). The similarity of RI deposition of H3.3 to epigenetic activation suggests that H3.3 is depositing at active, but not inactive rDNA arrays.

We could ascertain whether H3.3 deposits at sites of active or inactive rDNA arrays using an antibody to H3K9me, which decorates heterochromatin on all of the chromosome arms. At mitosis, the prominent labeling of $\mathrm{X}$-long by H3.3-GFP falls into a large gap in the H3K9me pattern (Fig. 6). Close examination of the X-short rDNA arrays that display H3.3-GFP staining after induction reveals that these spots always fall within a gap in the H3K9me pattern (Fig. 6B, inset). No such gaps were seen in uninduced cells, leading to the conclusion that gaps in H3K9me appeared as a consequence of induction, with concomitant deposition of H3.3 (Ahmad and Henikoff 2002c). This replacement process must have occurred within the 16-hour time window between induction by feeding and fixation of the cells. Recently Spector and coworkers (Janicki et al. 2004) confirmed and extended this observation by inducing a large array for transcription by RNA Polymerase II in a human cell line and showing that $\mathrm{H} 3 \mathrm{~K} 9$ me and HP1 disappear while $\mathrm{H} 3.3 \mathrm{ac}-$ cumulates to high levels during an approximately onehour period. Therefore, the replacement of heterochromatin by $\mathrm{H} 3.3$ appears to be a general process that can occur rapidly upon transcriptional induction.
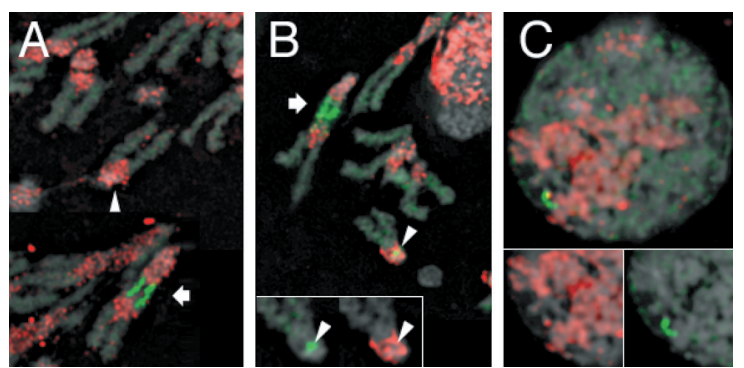

Figure 6. Replacement of H3K9me by H3.3 at rDNA arrays (Ahmad and Henikoff 2002c). (A) Mitotic figures from stationary phase cells show intense H3.3-GFP labeling (green) only on the large rDNA array on the elongated X-long chromosome (arrow), but not on the X-short chromosomes (arrowhead), which also carry rDNA genes. $(B)$ Mitotic figures from induced cells show H3.3-GFP labeling on both X-long and X-short chromosomes. (Inset) An enlargement of the proximal part of X-short. Wherever H3.3-GFP is present, there is a gap in heterochromatin (antibody to H3K9me, red). (C) RI H3.3-GFP labeling shows little overlap with heterochromatin in interphase nuclei. DNA staining (DAPI) is in gray. (Insets) Separated channels. (Reprinted, with permission, from Ahmad and Henikoff 2002c [@Elsevier].) 


\section{H3.3 IS ENRICHED IN “ACTIVE" LYSINE MODIFICATIONS}

If H3.3 marks active chromatin, then it should be enriched in covalent modifications that have been correlated with the active state. To test this, we fractionated histone 3 forms from $K c$ cells using reverse-phase high performance liquid chromatography (HPLC) (McKittrick et al. 2004), which had previously been used by Waterborg to resolve alfalfa H3.1 (the plant RC form) and H3.2 (the RI form) (Waterborg 1990). When acid-extracted nuclear proteins were chromatographed, histone 3 resolved into two peaks (Fig. 7). After ArgC protease digestion of HPLC fractions, we performed mass spectrometry. Based on the encoded difference at position 31, which is alanine in $\mathrm{H} 3$ and serine in $\mathrm{H} 3.3$, mass analysis of peptide $27-40$ identified the first peak as H3 and the second peak as H3.3 (McKittrick et al. 2004).

Repeated measurements of relative peak heights revealed that $\mathrm{H} 3.3$ accounts for $24 \pm 3 \%$ of bulk histone 3 in $K c$ cells. This percentage is not significantly different from the fraction (26\%) of the Drosophila genome that is transcribed in $K c$ cells ([176 Mb total genomic DNA - 59 $\mathrm{Mb}$ heterochromatic $-49 \mathrm{Mb}$ intergenic $] / 176 \mathrm{Mb}$ total genomic [Hoskins et al. 2002; Misra et al. 2002] $=0.386$, multiplied by the fraction [68\%] of genes that are transcribed in $K c$ cells $[0.39 \times 0.68=0.26$ ] [Schübeler et al. 2002]). Therefore, H3.3 is sufficiently abundant to package all actively transcribed regions of the Drosophila genome.

The separation of $\mathrm{H} 3$ and $\mathrm{H} 3.3$ allowed us to ask whether they show differences in covalent modifications. To do this, we performed mass analysis of ArgC-digested $\mathrm{H} 3$ and $\mathrm{H} 3.3$ peptides using liquid chromatography with electrospray ionization mass spectrometry (LC/ESI MS)

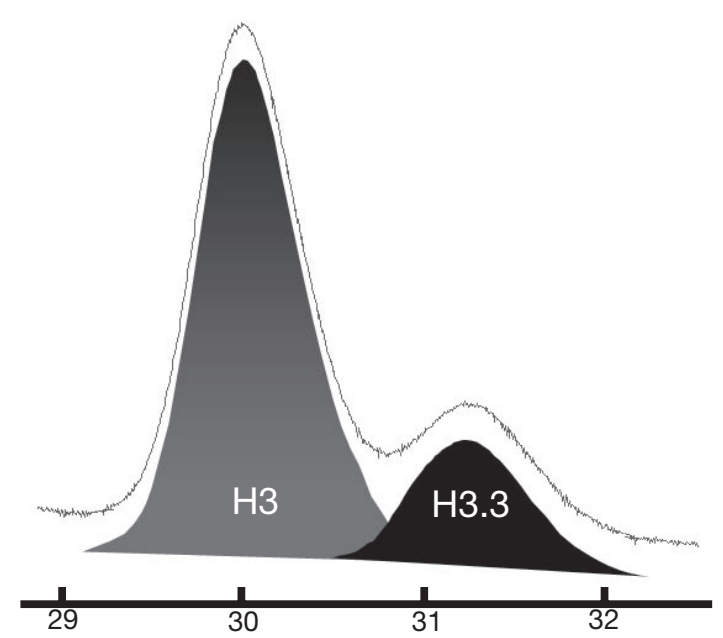

Figure 7. Abundance of H3.3 in a Drosophila cell line (McKittrick et al. 2004). Acid-extracted histones from $K c$ cells were injected onto a reverse-phase HPLC column in 30\% acetonitrile $0.1 \%$ trifluoroacetic acid. All histone 3 eluted in the two peaks shown here, and the peaks were determined to be composed of pure histone 3 by SDS PAGE and Coomassie staining. The relative abundance of the two peaks was determined by integrating the area under each curve (gray and black areas) and averaging over multiple measurements. and tandem mass spectrometry (McKittrick et al. 2004). We also measured modifications on H3 and H3.3 using modification-specific antibodies, assaying on western and slot blots and by enzyme-linked immunoabsorbent assays (ELISA). In all, we detected methyl and acetyl group on nine lysines (positions 4, 9, 14, 18, 23, 28, 36, 37 , and 79). All of these modifications are of residues that are identical between $\mathrm{H} 3$ and $\mathrm{H} 3.3$, and in no case did we detect a modification on one variant by LC/ESI MS but not on the other. However, there were numerous quantitative differences between $\mathrm{H} 3$ and $\mathrm{H} 3.3$ in the relative abundance of modifications. Relative to H3, H3.3 is enriched twofold to fivefold in di- and tri-methyl lysine 4, mono- and di-methyl lysine 79, and acetyl lysines 9, 14, and $23+28$, whereas H3 is enriched in dimethyl lysine 9 (Table 1). In general, our results on Drosophila Kc cells correspond well to those reported for alfalfa H3.1 and H3.2 by Waterborg (1990), who assayed modifications using Edman degradation analysis (Table 1). In both cases, hyperacetylation of tail lysines and enrichment of methylated K4 are found for the RI form and enrichment of dimethylated $\mathrm{K} 9$ is found for the RC forms of histone 3. Therefore, differences between RC and RI forms of histone 3 in the relative abundances of histone modifications are consistent between plants and animals. Lysine modifications are also highly abundant in plants and animals, with usually only a minority of each lysine residue on one or the other form of histone 3 remaining unmodified when measured in bulk (Waterborg 1990; McKittrick et al. 2004).

Table 1. Differential Enrichment of Modifications on Alternative Forms of Histone 3

\begin{tabular}{|c|c|c|c|c|c|}
\hline \multirow[b]{2}{*}{ Residue } & \multirow[b]{2}{*}{ Modification } & \multicolumn{2}{|c|}{ Drosophila $^{\mathrm{a}}$} & \multicolumn{2}{|c|}{ Alfalfa ${ }^{b}$} \\
\hline & & $\begin{array}{l}\mathrm{H} 3 \\
(\mathrm{RC})\end{array}$ & $\begin{array}{c}\text { H3.3 } \\
(\mathrm{RI})\end{array}$ & $\begin{array}{l}\text { H3.1 } \\
\text { (RC) }\end{array}$ & $\begin{array}{c}\text { H3.2 } \\
\text { (RI) }\end{array}$ \\
\hline \multirow[t]{3}{*}{$\overline{\mathrm{K} 4}$} & monomethyl & + & + & + & ++ \\
\hline & dimethyl & + & ++ & + & ++ \\
\hline & trimethyl & + & ++ & + & + \\
\hline \multirow[t]{3}{*}{ K9 } & monomethyl & + & + & ++ & + \\
\hline & $\begin{array}{l}\text { dimethyl } \\
\text { trimethyl }\end{array}$ & ++ & + & ++ & $\begin{array}{l}+ \\
++\end{array}$ \\
\hline & acetyl & + & ++ & & + \\
\hline \multirow[t]{4}{*}{ K14 } & monomethyl & + & + & & \\
\hline & dimethyl & + & + & & \\
\hline & trimethyl & & & + & + \\
\hline & acetyl & + & ++ & + & ++ \\
\hline $\mathrm{K} 18+\mathrm{K} 23$ & acetyl & + & ++ & + & ++ \\
\hline \multirow[t]{4}{*}{$\mathrm{K} 27$} & monomethyl & + & + & + & + \\
\hline & dimethyl & + & + & ++ & \\
\hline & trimethyl & + & + & + & + \\
\hline & acetyl & & & + & + \\
\hline \multirow[t]{2}{*}{ K36 } & monomethyl & + & + & & \\
\hline & dimethyl & + & + & & \\
\hline \multirow[t]{2}{*}{ K37 } & monomethyl & + & + & & \\
\hline & dimethyl & + & + & & \\
\hline \multirow[t]{2}{*}{ K79 } & monomethyl & + & ++ & & \\
\hline & dimethyl & + & ++ & & \\
\hline
\end{tabular}

+ , observed; ++ , relatively enriched.

a Determined by LC/ESI or tandem MS, ELISA, slot blot, and/or western blot analysis (McKittrick et al. 2004).

betermined by Edman analysis (Waterborg 1990). 
The modifications that are enriched on $\mathrm{H} 3.3$ relative to H3 are the same ones that many studies have shown to be enriched in active chromatin (Turner 2002), whereas dimethyl $\mathrm{K} 9$, which is enriched on $\mathrm{H} 3$ relative to $\mathrm{H} 3.3$, marks heterochromatin (e.g., Fig. 6). This biochemical correspondence strengthens our conclusions based on cytological observations that $\mathrm{H} 3.3$ marks active chromatin and $\mathrm{H} 3$ marks silent chromatin. Further support for this two-state model comes from a genome-wide chromatin immunoprecipitation analysis showing that actively transcribed genes are consistently enriched in acetylated $\mathrm{H} 3$ and $\mathrm{H} 4$ and in methylated H3K4 and H3K79 in Kc cells in an all-or-none fashion (Schübeler et al. 2004). Taken together, these observations suggest that histones are primarily modified as part of the nucleosome assembly process (Vermaak et al. 2003; McKittrick et al. 2004; Workman and Abmayr 2004). Modification enzymes would travel along with the RC and RI assembly complexes, acting just before, during, or just after assembly of the H3-H4 units into core nucleosomes. Replication and transcription are spatially and temporally separate processes that use chemically distinct substrates, and the same appears to be the case for their accompanying nucleosome assembly and modification complexes.

\section{RI NUCLEOSOME ASSEMBLY AND CHROMATIN INHERITANCE}

The relative abundance of covalent modifications that are associated with active chromatin on H3.3 may have profound implications for the relationship between chromatin and transcription in general. The assembly of $\mathrm{H} 3.3-\mathrm{H} 4$ at active, but not inactive, loci throughout the cell cycle (Fig. 4) and the sufficient abundance of H3.3 to package essentially all active chromatin (Fig. 7) suggest that RI assembly is a transcription-coupled process that results in the continual turnover of nucleosomes. RNA polymerase transit, together with associated chromatin remodeling machines (Kingston and Narlikar 1999), might provide the force to expel a nucleosome, either removing it entirely or exchanging it with new $\mathrm{H} 3.3-\mathrm{H} 4$ in a stepwise fashion (Henikoff et al. 2004). As a result, transcription units would become fully packaged in H3.3-containing nucleosomes over the course of a cell cycle, resulting in enrichment of histone modifications known to be associated with active chromatin. Genes would be inactivated by recruitment of histone deacetylases and H3K9 methyltransferases (Richards and Elgin 2002), which would act on H3.3-containing nucleosomes to promote a silent conformation.

Each round of cell division deposits H3-containing nucleosomes, which are relatively deficient in modifications associated with active chromatin. This is likely to be important for the majority of the genome, which must be kept in a transcriptionally silent and condensed state in typical higher eukaryotic cells. At transcriptionally active regions, $\mathrm{RC}$ assembly would cause a factor-of- 2 dilution of old H3.3-containing nucleosomes with those containing H3 (Fig. 8). But an abundance of active modifications on the remaining H3.3-containing nucleo-

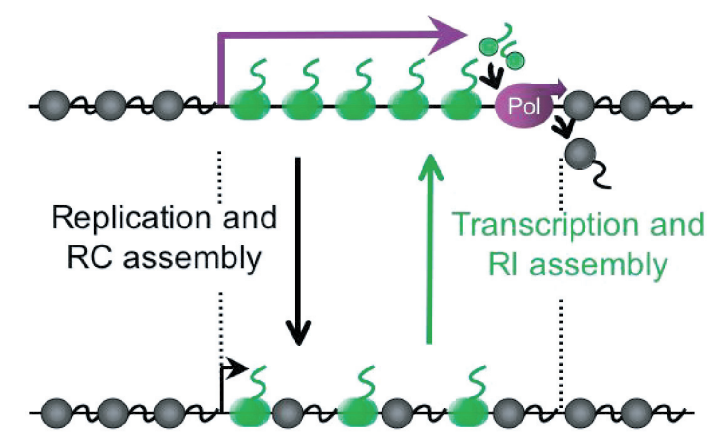

Figure 8. Model for chromatin inheritance (Henikoff et al. 2004). During active transcription, H3.3 is continually inserted, replacing $\mathrm{H} 3$ from the previous replication event. In this way, the DNA transited by RNA polymerases (magenta arrow) defines the boundaries for regions of active chromatin. Nucleosomes with histone tails that lack active modifications (gray circles) bind avidly to DNA and neighboring nucleosomes. Nucleosomes with active modifications are more mobile (depicted as blurry green circles) and their tails do not package chromatin tightly as a result of acetylations of lysines and other modifications enriched on H3.3. During DNA replication, H3containing nucleosomes are deposited, diluting H3.3-containing nucleosomes by half, only partially reducing mobility in the transcribed region compared to the rest of the genome. The presence of these mobile nucleosomes would allow transcription to proceed.

somes could be sufficient to maintain the transcriptional process.

There are several ways in which the presence of H3.3containing nucleosomes might open chromatin for transcription. For example, the more acetylated tails of H3.3 might reduce contact with linker DNA, thus allowing the DNA to be unwrapped for transcription during RNA polymerase transit (van Holde et al. 1992). In any case, the presence of some H3.3 in chromatin may be sufficient to maintain a positive feedback loop, where ongoing transcription-coupled replacement of H3- with H3.3-containing nucleosomes returns the active gene to a highly acetylated H3.3-enriched state during the remainder of the cell cycle (Fig. 8). In this way, the process of RI nucleosome assembly can resolve the enigma of chromatin inheritance by restoring activating epigenetic signals following replication over a precisely defined interval, the span of the transcription unit (Ahmad and Henikoff 2002b).

\section{CONCLUSIONS}

Despite tremendous progress in recent years in understanding the details of eukaryotic transcription and chromatin enzymology, we are just beginning to understand the molecular mechanisms that are responsible for PEV and developmental memory. Here we have described evidence that leads to surprisingly simple explanations for these phenomena, based on what we have learned about the dynamic behavior and assembly of nucleosomes. We find that the classical two-state model of active and silent chromatin based on nuclease accessibility (Weintraub and Groudine 1976) holds up remarkably well. The active chromatin state would be perpetuated despite the dilution 
of epigenetic information after DNA replication by the coupling of RI assembly to transcription.

Other processes that act on chromatin, including the mobilization of nucleosomes by remodeling complexes, the covalent modifications of histone tails, and the movement of polymerases, might also regulate the dynamic behavior of nucleosomes. Nucleosome assembly complexes that are specific for histone variants provide other means of affecting chromatin dynamics by altering the composition of nucleosomes (Krogan et al. 2003; Mizuguchi et al. 2003; Kobor et al. 2004; Tagami et al. 2004). Whereas $\mathrm{RC}$ deposition of nucleosomes behind the replication fork would assemble chromatin into a relatively immobile default state, the small fraction of a higher eukaryotic genome that is exposed for transcription is subject to RI assembly, which is potentially a gene-regulatory process. So despite differentiation of nucleosomes resulting from histone modifications, binding of chromatin-associated proteins, and incorporation of histone variants, the coupling of these events to assembly of nucleosomes at replication forks and transcription units provides a fundamentally simple framework for understanding epigenetic processes.

\section{ACKNOWLEDGMENTS}

We thank Danielle Vermaak, Jim Smothers, Harmit Malik, Také Furuyama, Yamini Dalal, and Yoshiko Mito for helpful discussions. This work was supported by the Howard Hughes Medical Institute.

\section{REFERENCES}

Ahmad K. and Henikoff S. 2001a. Centromeres are specialized replication domains in heterochromatin. J. Cell Biol. 153: 101. . 2001b. Modulation of a transcription factor counteracts heterochromatic gene silencing in Drosophila. Cell 104: 839. .2002a. Epigenetic consequences of nucleosome dynamics. Cell 111: 281 .

. 2002b. Histone H3 variants specify modes of chromatin assembly. Proc. Natl. Acad. Sci. (suppl. 4) 99: 16477.

$.2002 \mathrm{c}$. The histone variant H3.3 marks active chromatin by replication-independent nucleosome assembly. Mol. Cell 9: 1191

Cheutin T., McNairn A.J., Jenuwein T., Gilbert D.M., Singh P.B., and Misteli T. 2003. Maintenance of stable heterochromatin domains by dynamic HP1 binding. Science 299: 721 .

Dillon N. and Festenstein R. 2002. Unravelling heterochromatin: Competition between positive and negative factors regulates accessibility. Trends Genet. 18: 252.

Dorer D.R. and Henikoff S. 1994. Expansions of transgene repeats cause heterochromatin formation and gene silencing in Drosophila. Cell 77: 993.

Echalier G. 1997. Drosophila cells in culture. Academic Press, New York.

Festenstein R., Pagakis S.N., Hiragami K., Lyon D., Verreault A., Sekkali B., and Kioussis D. 2003. Modulation of Heterochromatin Protein 1 dynamics in primary mammalian cells. Science 299: 719.

Grewal S.I. and Elgin S.C. 2002. Heterochromatin: New possibilities for the inheritance of structure. Curr. Opin. Genet. Dev. 12: 178.

Henikoff S., Furuyama T., and Ahmad A. 2004. Histone variants, nucleosome assembly and epigenetic inheritance. Trends Genet. 20: 320.

Hoskins R.A., Smith C.D., Carlson J.W., Carvalho A.B., Halpern A., Kaminker J.S., Kennedy C., Mungall C.J., Sulli- van B.A., Sutton G.G., Yasuhara J.C., Wakimoto B.T., Myers E.W., Celniker S.E., Rubin G.M., and Karpen G.H. 2002. Heterochromatic sequences in a Drosophila whole-genome shotgun assembly. Genome Biol. 3: RESEARCH0085.

Janicki S.M., Tsukamoto T., Salghetti S.E., Tansey W.P., Sachidanandam R., Prasanth K.V., Ried T., Shav-Tal Y., Bertrand E., Singer R.H., and Spector D.L. 2004. From silencing to gene expression: Real-time analysis in single cells. Cell 116: 683.

Jenuwein T. and Allis C.D. 2001. Translating the histone code. Science 293: 1074

Kingston R.E. and Narlikar G.J. 1999. ATP-dependent remodeling and acetylation as regulators of chromatin fluidity. Genes Dev. 13: 2339.

Kobor M.S., Venkatasubrahmanyam S., Meneghini M.D., Gin J.W., Jennings J.L., Link A.J., Madhani H.D., and Rine J. 2004. A protein complex containing the conserved Swi2/Snf2-related ATPase Swr1p deposits histone variant H2A.Z into euchromatin. PLoS Biol. 2: E131.

Krogan N.J., Keogh M.C., Datta N., Sawa C., Ryan O.W., Ding H., Haw R.A., Pootoolal J., Tong A., Canadien V., Richards D.P., Wu X., Emili A., Hughes T.R., Buratowski S., and Greenblatt J.F. 2003. A Snf2 family ATPase complex required for recruitment of the histone H2A variant Htz1. Mol. Cell 12: 1565.

Leffak I.M., Grainger R., and Weintraub H. 1977. Conservative assembly and segregation of nucleosomal histones. Cell 12: 837.

Leonhardt H., Page A.W., Weier H.U., and Bestor T.H. 1992. A targeting sequence directs DNA methyltransferase to sites of DNA replication in mammalian nuclei. Cell 71: 865 .

Luger K., Mader A.W., Richmond R.K., Sargent D.F., and Richmond T.J. 1997. Crystal structure of the nucleosome core particle at $2.8 \AA$ resolution. Nature 389: 251.

Malik H.S. and Henikoff S. 2003. Phylogenomics of the nucleosome. Nat. Struct. Biol. 10: 882.

McKittrick E., Gafken P.R., Ahmad K., and Henikoff S. 2004. Histone H3.3 is enriched in covalent modifications associated with active chromatin. Proc. Natl. Acad. Sci. 101: 1525.

Mello J.A. and Almouzni G. 2001. The ins and outs of nucleosome assembly. Curr. Opin. Genet. Dev. 11: 136.

Misra S., Crosby M.A., Mungall C.J., Matthews B.B., Campbell K.S., Hradecky P., Huang Y., Kaminker J.S., Millburn G.H., et al. 2002. Annotation of the Drosophila melanogaster euchromatic genome: A systematic review. Genome Biol. 3: RESEARCH0083.

Mizuguchi G., Shen X., Landry J., Wu W.H., Sen S., and Wu C. 2003. ATP-driven exchange of histone H2AZ variant catalyzed by SWR1 chromatin remodeling complex. Science 303: 343 .

Muller H.J. 1930. Types of visible variations induced by X-rays in Drosophila. J. Genet. 22: 299.

Pikaard C.S. 2000. The epigenetics of nucleolar dominance. Trends Genet. 16: 495.

Polach K.J. and Widom J. 1995. Mechanism of protein access to specific DNA sequences in chromatin: A dynamic equilibrium model for gene regulation. J. Mol. Biol. 254: 130 .

Richards E.J. and Elgin S.C. 2002. Epigenetic codes for heterochromatin formation and silencing: Rounding up the usual suspects. Cell 108: 489.

Schübeler D., Scalzo D., Kooperberg C., van Steensel B., Delrow J., and Groudine M. 2002. Genome-wide DNA replication profile for Drosophila melanogaster: A link between transcription and replication timing. Nat. Genet. 32: 438.

Schübeler D., MacAlpine D.M., Scalzo D., Wirbelauer C., Kooperberg C., van Leeuwen F., Gottschling D.E., O’Neill L.P., Turner B.M., Delrow J., Bell S.P., and Groudine M. 2004. The histone modification pattern of active genes revealed through genome-wide chromatin analysis of a higher eukaryote. Genes Dev. 18: 1263.

Shelby R.D., Monier K., and Sullivan K.F. 2000. Chromatin assembly at kinetochores is uncoupled from DNA replication. $J$. Cell Biol. 151: 1113.

Spofford J.B. 1976. Position-effect variegation in Drosophila. In Genetics and biology of Drosophila (ed. M. Ashburner and E. Novitski), p. 955. Academic Press, London. 
Tagami H., Ray-Gallet D., Almouzni G., and Nakatani Y. 2004. Histone H3.1 and H3.3 complexes mediate nucleosome assembly pathways dependent or independent of DNA synthesis. Cell 116: 51.

Turner B.M. 2002. Cellular memory and the histone code. Cell 111: 285 .

van Holde K.E., Lohr D.E., and Robert C. 1992. What happens to nucleosomes during transcription? J. Biol. Chem. 267: 2837.

Vermaak D., Ahmad K., and Henikoff S. 2003. Maintenance of chromatin states: An open-and-shut case. Curr. Opin. Cell Biol. 15: 266.

Waterborg J.H. 1990. Sequence analysis of acetylation and methylation in two histone $\mathrm{H} 3$ variants of alfalfa. J. Biol. Chem. 265: 17157.

. 1993. Histone synthesis and turnover in alfalfa: Fast loss of highly acetylated replacement histone variant H3.2. J. Biol. Chem. 268: 4912.
Weiler K.S. and Wakimoto B.T. 1995. Heterochromatin and gene expression in Drosophila. Annu. Rev. Genet. 29: 577.

Weintraub H. and Groudine M. 1976. Chromosomal subunits in active genes have an altered conformation. Science 193: 848.

Workman J.L. and Abmayr S.M. 2004. Histone H3 variants and modifications on transcribed genes. Proc. Natl. Acad. Sci. 101: 1429

Yamasu K. and Senshu T. 1990. Conservative segregation of tetrameric units of $\mathrm{H} 3$ and $\mathrm{H} 4$ histones during nucleosome replication. J. Biochem. 107: 15.

Yu L. and Gorovsky M.A. 1997. Constitutive expression, not a particular primary sequence, is the important feature of the $\mathrm{H} 3$ replacement variant hv2 in Tetrahymena thermophila. Mol. Cell. Biol. 17: 6303.

Zheng C. and Hayes J.J. 2003. Structures and interactions of the core histone tail domains. Biopolymers 68: 539. 


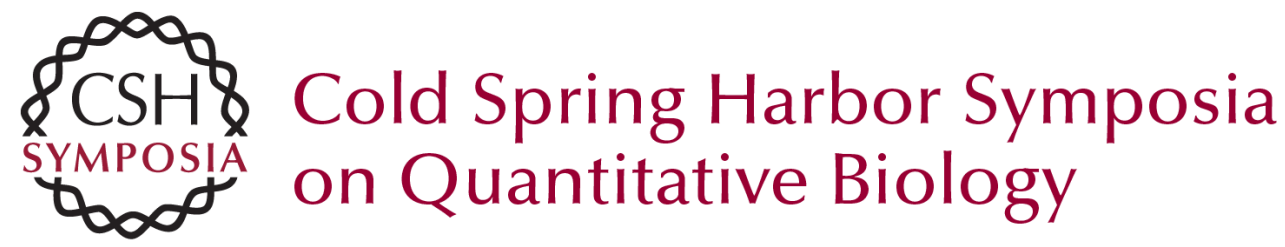

\section{Epigenetics, Histone $\mathrm{H} 3$ Variants, and the Inheritance of Chromatin States}

S. HENIKOFF, E. MCKITTRICK and K. AHMAD

Cold Spring Harb Symp Quant Biol 2004 69: 235-244

Access the most recent version at doi:10.1101/sqb.2004.69.235

References This article cites 42 articles, 15 of which can be accessed free at: http://symposium.cshlp.org/content/69/235.full.html\#ref-list-1

License

Email Alerting Receive free email alerts when new articles cite this article - sign up in Service the box at the top right corner of the article or click here. 